\title{
Solving Linear and Nonlinear Fractional Differential Equations Using Spline Functions
}

\author{
Adel Al-Rabtah, ${ }^{1}$ Shaher Momani, ${ }^{2}$ and Mohamed A. Ramadan ${ }^{3}$ \\ ${ }^{1}$ Department of Mathematics and Statistics, Mutah University, P.O. Box 7, Al-Karak, Jordan \\ ${ }^{2}$ Department of Mathematics and Statistics, University of Jordan, Amman 11942, Jordan \\ ${ }^{3}$ Department of Mathematics, Faculty of Science, Minoufiya University, Shebeen El Koom, \\ Minoufiya, Egypt \\ Correspondence should be addressed to Adel Al-Rabtah, adel_alrabtah@yahoo.com
}

Received 29 November 2011; Revised 23 December 2011; Accepted 26 December 2011

Academic Editor: Muhammad Aslam Noor

Copyright (C) 2012 Adel Al-Rabtah et al. This is an open access article distributed under the Creative Commons Attribution License, which permits unrestricted use, distribution, and reproduction in any medium, provided the original work is properly cited.

\begin{abstract}
Suitable spline functions of polynomial form are derived and used to solve linear and nonlinear fractional differential equations. The proposed method is applicable for $0<\alpha \leq 1$ and $\alpha \geq 1$, where $\alpha$ denotes the order of the fractional derivative in the Caputo sense. The results obtained are in good agreement with the exact analytical solutions and the numerical results presented elsewhere. Results also show that the technique introduced here is robust and easy to apply.
\end{abstract}

\section{Introduction}

Mathematical modelling of complex processes is a major challenge for contemporary scientists. In contrast to simple classical systems, where the theory of integer-order differential equations is sufficient to describe their dynamics, fractional derivatives provide an excellent instrument for the description of memory and hereditary properties of various complex materials and systems. Therefore, the number of scientific and engineering problems involving fractional derivatives is already very large and still growing and perhaps the fractional calculus (i.e., derivatives and integrals of any real or complex order) will be the calculus of the twenty-first century [1-6].

The analytic results on the existence and uniqueness of solutions to the fractional differential equations have been investigated by many authors (see, e.g., $[7,8]$ ). During the last decades, several numerical and analytical methods have been proposed in the literature to solve fractional differential equations. The most commonly used ones are fractional difference method $[9,10]$, Adomian decomposition method [11], variational iteration method 
$[12,13]$, and Adams-Bashforth-Moulton method [14-16]. Recently, Lagrange multiplier method and the homotopy perturbation method are used to numerically solve multiorder fractional differential equations, see [17].

In view of successful application of spline functions of polynomial form in system analysis [18], delay differential equations [19], and delay differential equations of fractional order [20], we hold that it should be applicable to solve linear and nonlinear fractional-order systems.

In the present paper, we intend to extend the application of the spline functions of polynomial form to solve the fractional differential equations:

$$
y^{\alpha}(x)=f(x, y(x)), \quad y(0)=y_{0},
$$

where $\alpha$ denotes the order of the fractional derivative in the Caputo sense.

\section{The Spline Function Method of Polynomial Form}

Recently, many studies were devoted to the problems of approximate solutions of system ordinary as well as delay differential equations by spline functions [18-21]. Micula et al. [18] considered the following system:

$$
\begin{array}{ll}
y^{\prime}(x)=f_{1}(x, y, z), & y\left(x_{0}\right)=y_{0}, \\
z^{\prime}(x)=f_{2}(x, y, z), & z\left(x_{0}\right)=z_{0},
\end{array}
$$

where $f_{1}, f_{2} \in C^{r}[0,1] \times \mathbb{R} \times \mathbb{R},(x, y, z) \in[0,1] \times \mathbb{R} \times \mathbb{R}$. They assumed that the functions $f_{i}^{(q)}, i=1,2$, and $q=0,1, \ldots, r$ satisfy the Lipschitz condition of the form

$$
\left|f_{i}^{(q)}\left(x, y_{1}, z_{1}\right)-f_{i}^{(q)}\left(x, y_{2}, z_{2}\right)\right| \leq L_{i}\left\{\left|y_{1}-y_{2}\right|+\left|z_{1}-z_{2}\right|\right\},
$$

with constant $L_{i}$ for all $\left(x, y_{i}, z_{i}\right) \in[0,1] \times \mathbb{R} \times \mathbb{R}$. In their paper, they presented investigations to the extension of the spline functions form for approximating the solution of the system of initial value problems (2.1), with unique solutions $y=y(x)$ and $z=z(x)$.

The spline functions $S_{\Delta}$ and $\tilde{S}_{\Delta}$ to approximate $y=y(x)$ and $z=z(x)$, respectively, are defined in polynomial form as

$$
\begin{aligned}
& S_{\Delta}(x)=S_{k}(x)=S_{k-1}\left(x_{k}\right)+\sum_{i=0}^{r} f_{1}^{(i)}\left(x_{k}, S_{k-1}\left(x_{k}\right), \widetilde{S}_{k-1}\left(x_{k}\right)\right) \frac{\left(x-x_{k}\right)^{i+1}}{(i+1) !}, \\
& \widetilde{S}_{\Delta}(x)=\widetilde{S}_{k}(x)=\widetilde{S}_{k-1}\left(x_{k}\right)+\sum_{i=0}^{r} f_{2}^{(i)}\left(x_{k}, S_{k-1}\left(x_{k}\right), \widetilde{S}_{k-1}\left(x_{k}\right)\right) \frac{\left(x-x_{k}\right)^{i+1}}{(i+1) !}
\end{aligned}
$$

for $x \in\left[x_{k}, x_{k+1}\right], k=0,1, \ldots, n-1, S_{-1}\left(x_{0}\right)=y_{0}, \widetilde{S}_{-1}\left(x_{0}\right)=z_{0}$. 
Ramadan introduced in [19] the solution of the first-order delay differential equation of the form:

$$
\begin{gathered}
y^{\prime}(x)=f(x, y(x), y(g(x))), \quad a \leq x \leq b, \\
y(a)=y_{0}, \quad y(x)=\Phi(x), \quad x \in\left[a^{*}, a\right], a^{*}<0, a^{*}=\inf \{g(x): x \in[a, b]\},
\end{gathered}
$$

using the spline functions of the polynomial form, defined as

$$
S_{\Delta}(x)=S_{k}(x)=S_{k-1}\left(x_{k}\right)+\sum_{i=0}^{r} M_{k}^{(i)} \frac{\left(x-x_{k}\right)^{i+1}}{(i+1) !},
$$

where $M_{k}^{(i)}=f^{(i)}\left(x_{k}, S_{k-1}\left(x_{k}\right), S_{k-1}\left(g\left(x_{k}\right)\right)\right)$, with $S_{-1}\left(x_{0}\right)=y_{0}, S_{-1}\left(g\left(x_{0}\right)\right)=\Phi\left(g\left(x_{0}\right)\right)$.

Ramadan in [21] considered the system of the initial value problem

$$
\begin{array}{ll}
y^{\prime \prime \prime}(x)=f_{1}\left(x, y, y^{\prime}, z, z^{\prime}\right), & y^{(i)}\left(x_{0}\right)=y_{0}^{(i)} \\
z^{\prime \prime \prime}(x)=f_{2}\left(x, y, y^{\prime}, z, z^{\prime}\right), & z^{(i)}\left(x_{0}\right)=z_{0}^{(i)}
\end{array}
$$

where $f_{1}, f_{2} \in C^{r}\left([0,1] \times \mathbb{R}^{(4)}\right), i=0,1,2$. The method in their work is based on polynomial splines, to approximate the solutions of the system.

Ramadan et al. [20] presented an extension and generalization of the polynomial spline functions used in the case of [19], to approximate the solution of the first delay differential equation and to investigate the solution of the fractional ordinary differential equation given by

$$
\begin{gathered}
y^{(\alpha)}(x)=f(x, y(x)), \quad a \leq x \leq b, \\
y(a)=y_{0}, \quad \alpha>1 .
\end{gathered}
$$

The formulation of the method presented in [20] is based on the fractional generalization of Taylor's theorem [22], which is presented in the following theorem

Theorem 2.1. Let $\alpha>0, n \in \mathbb{Z}^{+}$, and $f(x) \in C^{[\alpha]+n+1}([a, b])$. Then

$$
f(x)=\sum_{k=-n}^{n-1} \frac{D_{a+}^{\alpha+k} f\left(x_{0}\right)}{\Gamma(\alpha+k+1)}\left(x-x_{0}\right)^{\alpha+k}+R_{n}(x),
$$

for all $a \leq x_{0}<x \leq b$, where

$$
R_{n}(x)=J_{a+}^{\alpha+n} D_{a+}^{\alpha+n} f(x)
$$

is the remainder.

Error estimation and convergence analysis of the proposed method was presented by Ramadan et al. [20]. In their work, they used $\alpha>1$ and applied the method to solve two linear cases. 
The present paper is a sequel to this work [20], and we extend the application of spline functions method of polynomial form to a more general case incorporating nonlinearities as well. And we show that this analysis is also applicable in the case $0<\alpha \leq 1$, namely,

$$
\begin{gathered}
y^{(\alpha)}(x)=f(x, y(x)), \quad a \leq x \leq b, \\
y(a)=y_{0}, \quad \alpha>0 .
\end{gathered}
$$

Let $\Delta$ be a uniform partition to the interval $[a, b]$, defined by the nodes $\Delta: a=x_{0}<x_{1}<\cdots<$ $x_{k}<x_{k+1}<\cdots<x_{n}=b$, where $x_{k}=x_{0}+k h, k=0,1, \ldots, n$, and $h=(b-a) / n$.

Define the form of fractional spline function $S(x)$ of polynomial form approximating the exact solution $y$ by

$$
S_{\Delta}(x)=S_{k}(x)=S_{k-1}^{(\alpha-1)}\left(x_{k}\right) \frac{\left(x-x_{k}\right)^{\alpha-1}}{\Gamma(\alpha)}+\sum_{i=-n+1}^{r} M_{k}^{(\alpha+i-1)} \frac{\left(x-x_{k}\right)^{\alpha+i}}{\Gamma(\alpha+i+1)}, \quad n \in \mathbb{Z}^{+},
$$

where $M_{k}^{(\alpha)}=f^{(\alpha)}\left(x_{k}, S_{k-1}\left(x_{k}\right)\right)$, with $S_{-1}\left(x_{0}\right)=y_{0}$, for $x \in\left[x_{k}, x_{k+1}\right]$.

Definition 2.2. Let $\alpha \in \mathbb{R}_{+}$. The operator $J_{a}^{\alpha}$, defined on $L_{1}[a, b]$ by

$$
J_{a}^{\alpha} f(x):=\frac{1}{\Gamma(\alpha)} \int_{a}^{x} \frac{f(t)}{(x-t)^{1-\alpha}} d t
$$

for $a \leq x \leq b$, is called the Riemann-Lioville fractional integral operator of order $\alpha$.

For $\alpha=0$, we set $J_{a}^{0}:=I$, the identity operator.

Definition 2.3. The Caputo fractional derivative of $f(x)$, of order $\alpha>0$ with $a \geq 0$, is defined as

$$
\left(D_{a}^{\alpha} f\right)(x)=\left(J_{a}^{m-\alpha} f^{(m)}\right)(x):=\frac{1}{\Gamma(m-\alpha)} \int_{a}^{x} \frac{f^{(m)}(t)}{(x-t)^{\alpha+1-m}} d t
$$

for $m-1 \leq \alpha \leq m, m \in \mathbb{N}, x \geq a$, and $f(x) \in C_{-1}^{m}$.

The Riemann-Liouville fractional operators may be extended to hold for large values of $\alpha$, so we denote $\alpha=\lfloor\alpha\rfloor+\beta$, where $\lfloor\alpha\rfloor$ is the integer part of $\alpha$, and $\beta=\alpha-\lfloor\alpha\rfloor$, thus we give the following definition.

Definition 2.4. If $\alpha>0$ and $\alpha \notin \mathbb{N}$, then we define

$$
D_{a}^{\alpha} f=\frac{d^{\lfloor\alpha\rfloor}}{d x^{\lfloor\alpha\rfloor}} D_{a}^{\beta} f=\frac{d^{\lfloor\alpha\rfloor+1}}{d x^{\lfloor\alpha\rfloor+1}} J_{a}^{1-\beta} f
$$

thus

$$
\left(D_{a}^{\alpha} f\right)(x)=\frac{1}{\Gamma(m-\alpha)} \frac{d^{m}}{d x^{m}} \int_{a}^{x} \frac{f(t)}{(x-t)^{\alpha+1-m}} d t
$$


Table 1: Solution of Example 3.1 using $\alpha=0.5$ and $\alpha=0.75$.

\begin{tabular}{lcccc}
\hline$x$ & \multicolumn{2}{c}{$\alpha=0.5$} & \multicolumn{2}{c}{$\alpha=0.75$} \\
& Appr. solution & Absolute error & Appr. solution & Absolute error \\
\hline 0.0 & 0.0 & 0.0 & 0.0 & 0.0 \\
0.1 & 0.0 & $4.0000 \times 10^{-4}$ & 0.0 & $4.0000 \times 10^{-4}$ \\
0.2 & $-3.5668 \times 10^{-3}$ & $1.1668 \times 10^{-3}$ & $-2.8054 \times 10^{-3}$ & $4.0543 \times 10^{-4}$ \\
0.3 & $-5.6930 \times 10^{-3}$ & $2.9299 \times 10^{-4}$ & $-5.8753 \times 10^{-3}$ & $4.7533 \times 10^{-4}$ \\
0.4 & $-6.8508 \times 10^{-3}$ & $4.5080 \times 10^{-4}$ & $-6.7877 \times 10^{-3}$ & $3.8767 \times 10^{-4}$ \\
0.5 & $2.2930 \times 10^{-3}$ & $2.2930 \times 10^{-3}$ & $9.7776 \times 10^{-4}$ & $9.7776 \times 10^{-4}$ \\
0.6 & $2.8146 \times 10^{-2}$ & $6.5464 \times 10^{-3}$ & $2.6460 \times 10^{-2}$ & $4.8601 \times 10^{-3}$ \\
0.7 & $8.3911 \times 10^{-2}$ & $1.5311 \times 10^{-2}$ & $8.1759 \times 10^{-2}$ & $1.3159 \times 10^{-2}$ \\
0.8 & $1.8149 \times 10^{-1}$ & $2.7885 \times 10^{-2}$ & $1.8163 \times 10^{-1}$ & $2.8034 \times 10^{-2}$ \\
0.9 & $3.3755 \times 10^{-1}$ & $4.5954 \times 10^{-2}$ & $3.4348 \times 10^{-1}$ & $5.1882 \times 10^{-2}$ \\
1.0 & $5.6863 \times 10^{-1}$ & $6.8632 \times 10^{-2}$ & $5.8718 \times 10^{-1}$ & $8.7178 \times 10^{-2}$ \\
\hline
\end{tabular}

for any $f \in C^{m}([a, b])$, where $m=\lfloor\alpha\rfloor+1$. If, on the other hand, $\alpha<0$, then the definition becomes

$$
D_{a}^{\alpha} f=J_{a}^{-\alpha} f
$$

\section{Numerical Examples}

To demonstrate the effectiveness of this scheme, we consider two kinds of systems: one is linear and the other is nonlinear. These examples are considered because closed form solutions are available for them, or they have also been solved using other numerical schemes. This allows one to compare the results obtained using this scheme with the analytical solution or the solutions obtained using other schemes.

Example 3.1. Consider the fractional differential equation

$$
\mathrm{D}^{\alpha} y(x)=-y(x)+x^{4}-\frac{1}{2} x^{3}-\frac{3}{\Gamma(4-\alpha)} x^{3-\alpha}+\frac{24}{\Gamma(5-\alpha)} x^{4-\alpha}, \quad 0<\alpha \leq 1
$$

with initial condition $y(0)=0$.

The exact solution is

$$
y(x)=x^{4}-\frac{1}{2} x^{3}
$$

The numerical results obtained, for different values of $\alpha$, and for $0 \leq x \leq 1$, are shown in Table 1 and Table 2, together with absolute errors, to illustrate the accuracy of the spline method of polynomial form. Figure 1(a) for $\alpha=0.5$, Figure 1(b) for $\alpha=0.75$, Figure 1(c) for $\alpha=0.9$, and Figure $1(\mathrm{~d})$ for $\alpha=1.0$ show the approximate solutions compared to the exact solution, to better illustrate the accuracy of the method. 
Table 2: Solution of Example 3.1 using $\alpha=0.9$, and $\alpha=1$.

\begin{tabular}{lcccc}
\hline & \multicolumn{2}{c}{$\alpha=0.9$} & \multicolumn{2}{c}{$\alpha=1.0$} \\
$x$ & Appr. solution & Absolute error & Appr. solution & Absolute error \\
\hline 0.0 & 0.0 & 0.0 & 0 & 0.0 \\
0.1 & 0.0 & $4.0000 \times 10^{-4}$ & $-1.0000 \times 10^{-3}$ & $6.0000 \times 10^{-4}$ \\
0.2 & $-2.4593 \times 10^{-3}$ & $5.9337 \times 10^{-5}$ & $-2.9700 \times 10^{-3}$ & $5.7000 \times 10^{-4}$ \\
0.3 & $-5.4032 \times 10^{-3}$ & $3.1688 \times 10^{-6}$ & $-5.6338 \times 10^{-3}$ & $2.3383 \times 10^{-4}$ \\
0.4 & $-6.0963 \times 10^{-3}$ & $3.0367 \times 10^{-4}$ & $-5.9088 \times 10^{-3}$ & $4.9117 \times 10^{-4}$ \\
0.5 & $1.3284 \times 10^{-3}$ & $1.3284 \times 10^{-3}$ & $1.5404 \times 10^{-3}$ & $1.5404 \times 10^{-3}$ \\
0.6 & $2.5395 \times 10^{-2}$ & $3.7951 \times 10^{-3}$ & $2.4489 \times 10^{-2}$ & $2.8891 \times 10^{-3}$ \\
0.7 & $7.7426 \times 10^{-2}$ & $8.8255 \times 10^{-3}$ & $7.3107 \times 10^{-2}$ & $4.5075 \times 10^{-3}$ \\
0.8 & $1.7148 \times 10^{-1}$ & $1.7883 \times 10^{-2}$ & $1.5997 \times 10^{-1}$ & $6.3700 \times 10^{-3}$ \\
0.9 & $3.2435 \times 10^{-1}$ & $3.2755 \times 10^{-2}$ & $3.0005 \times 10^{-1}$ & $8.4533 \times 10^{-3}$ \\
1.0 & $5.5552 \times 10^{-1}$ & $5.5518 \times 10^{-2}$ & $5.1074 \times 10^{-1}$ & $1.0736 \times 10^{-2}$ \\
\hline
\end{tabular}

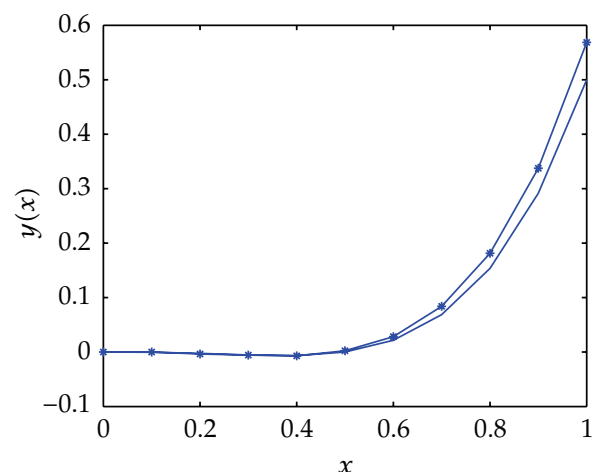

Exact solution $\rightarrow-\alpha=0.5$

(a) Solution at $\alpha=0.5$

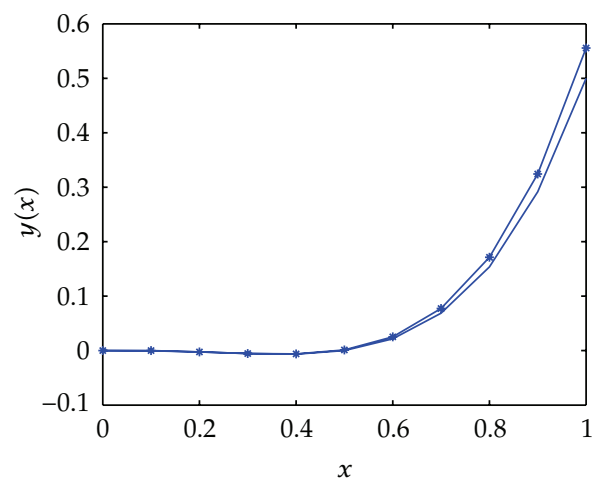

- Exact solution

$\rightarrow \alpha=0.9$

(c) Solution at $\alpha=0.9$

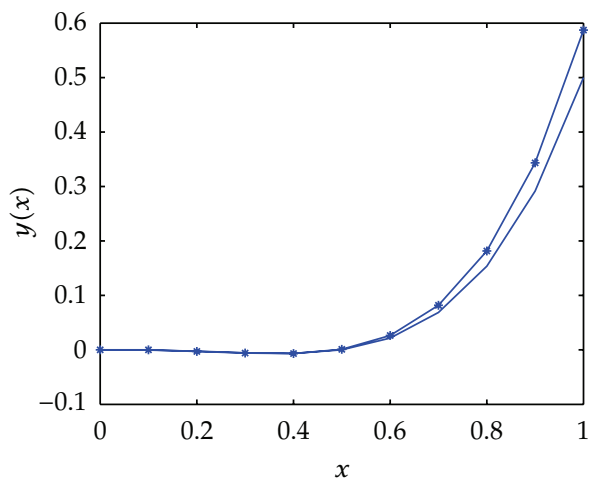

- Exact solution

* $\alpha=0.75$

(b) Solution at $\alpha=0.75$

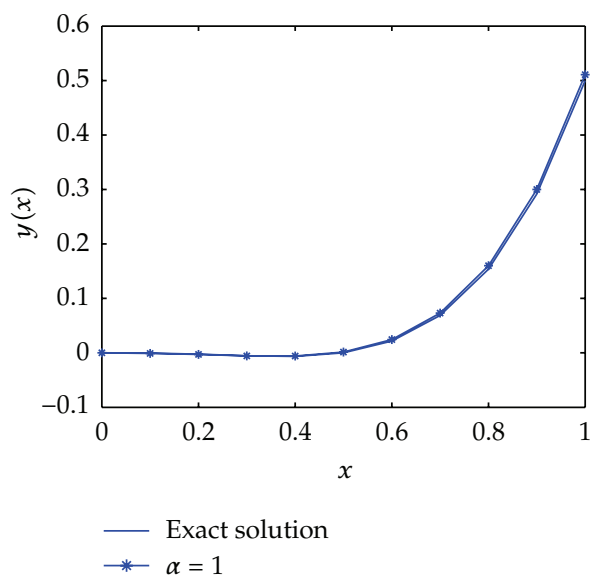

(d) Solution at $\alpha=1.0$

Figure 1: Approximate solutions for Example 3.1. 
Table 3: Solution of Example 3.2 using $\alpha=1.3, \alpha=1.1$, and $\alpha=1$.

\begin{tabular}{|c|c|c|c|c|}
\hline \multirow[b]{2}{*}{$x$} & \multirow{2}{*}{$\begin{array}{c}\alpha=1.3 \\
\text { Appr. solution }\end{array}$} & \multirow{2}{*}{$\begin{array}{c}\alpha=1.1 \\
\text { Appr. solution }\end{array}$} & \multicolumn{2}{|c|}{$\alpha=1$} \\
\hline & & & Appr. solution & Absolute error \\
\hline 0.0 & 0.00000 & 0.00000 & 0.00000 & 0.0 \\
\hline 0.5 & 0.32538 & 0.41045 & 0.45833 & $3.7838 \times 10^{-3}$ \\
\hline 1.0 & 0.52950 & 0.67714 & 0.76095 & $6.4768 \times 10^{-4}$ \\
\hline 1.5 & 0.63709 & 0.81882 & 0.90603 & $8.7977 \times 10^{-4}$ \\
\hline 2.0 & 0.68987 & 0.89089 & 0.96437 & $3.3837 \times 10^{-4}$ \\
\hline 2.5 & 0.71508 & 0.92755 & 0.98660 & $1.5476 \times 10^{-5}$ \\
\hline 3.0 & 0.72699 & 0.94629 & 0.99497 & $8.4583 \times 10^{-5}$ \\
\hline 3.5 & 0.73259 & 0.95590 & 0.99811 & $6.4645 \times 10^{-5}$ \\
\hline 4.0 & 0.73522 & 0.96084 & 0.99929 & $3.6906 \times 10^{-5}$ \\
\hline 4.5 & 0.73646 & 0.96339 & 0.99973 & $1.8574 \times 10^{-5}$ \\
\hline 5.0 & 0.73703 & 0.96470 & 0.99990 & $8.7168 \times 10^{-6}$ \\
\hline
\end{tabular}

Example 3.2. Consider the following fractional Riccati equation:

$$
\mathrm{D}^{\alpha} y(x)=-y(x)+1, \quad 1 \leq \alpha<2,
$$

subject to the initial condition $y(0)=0$.

The exact solution, when $\alpha=1$, is

$$
y(x)=\frac{e^{2 x}-1}{e^{2 x}+1}
$$

The numerical results obtained, for different values of $\alpha$, and for $0 \leq x \leq 5$, are shown in Table 3; the approximate solution for $\alpha=1$ is compared to the exact solution, to illustrate the accuracy of the spline method of polynomial form.

Example 3.3. Consider the following nonlinear fractional ordinary differential equation:

$$
\mathrm{D}^{\alpha} y(t)=A(1-y)^{4}, \quad A \in \mathbb{R}^{+}, 0<\alpha \leq 1,
$$

subject to the initial condition $y(0)=\beta$, where $\beta$ is a real constant.

This equation describes the cooling of a semi-infinite body by radiation, and the initial value problem has been solved numerically using the fractional difference method and Adomian decomposition method in [23].

The exact solution, when $\alpha=1, \beta=0$, and $A=1$, is

$$
y(t)=\frac{1+3 t-\left(1+6 t+9 t^{2}\right)^{1 / 3}}{1+3 t}
$$


Table 4: Solution of Example 3.3 using $\alpha=0.75, \alpha=0.9$, and $\alpha=1$.

\begin{tabular}{lcccc}
\hline$t$ & $\begin{array}{c}\alpha=0.75 \\
\text { Appr. solution }\end{array}$ & $\begin{array}{c}\alpha=0.9 \\
\text { Appr. solution }\end{array}$ & Appr. solution & Absolute error \\
\hline 0.0 & 0.00000 & 0.00000 & 0.00000 & 0.0 \\
0.1 & 0.14054 & 0.10265 & 0.08383 & $9.3660 \times 10^{-5}$ \\
0.2 & 0.21290 & 0.17219 & 0.14513 & $1.1397 \times 10^{-4}$ \\
0.3 & 0.25536 & 0.22294 & 0.19272 & $1.1162 \times 10^{-4}$ \\
0.4 & 0.28215 & 0.26188 & 0.23122 & $1.0297 \times 10^{-4}$ \\
0.5 & 0.29977 & 0.29284 & 0.26329 & $9.3223 \times 10^{-5}$ \\
0.6 & 0.31165 & 0.31809 & 0.29059 & $8.4033 \times 10^{-5}$ \\
0.7 & 0.31980 & 0.33910 & 0.31425 & $7.5850 \times 10^{-5}$ \\
0.8 & 0.32544 & 0.35683 & 0.33504 & $6.8710 \times 10^{-5}$ \\
0.9 & 0.32939 & 0.37199 & 0.35352 & $6.2520 \times 10^{-5}$ \\
1.0 & 0.33215 & 0.38508 & 0.37010 & $5.7152 \times 10^{-5}$ \\
\hline
\end{tabular}

In this paper, we use the spline function method of polynomial form, to solve the fractional differential equation (3.5), together with $y(0)=\beta=0$ and $A=1$. Table 4 shows the approximate solutions for (3.5) obtained for different values of $\alpha$ using the spline method of polynomial form. From the numerical results in Table 4, it is clear that the approximate solutions are in high agreement with the exact solutions, when $\alpha=1$, and with those obtained in [23] using the fractional difference method and Adomian decomposition method.

\section{Conclusions}

In this paper, we investigated the possibility of extending and generalizing the spline functions of polynomial fractional form given in Ramadan et al. [20] to be applicable for $\alpha \geq 1$ as well as the case $0<\alpha \leq 1$. The method is tested by considering three test problems for three fractional ordinary differential equations. Two examples are of fractional order $\alpha$, $0<\alpha \leq 1$ (Example 3.1 and 3), while Example 3.2 is of fractional order $\alpha$ where $1 \leq \alpha<2$. The obtained numerical results are in good agreement with the exact analytical solutions.

\section{References}

[1] J. S. Leszczynski, An Itorduction to Fractional Mechanics, Czestochowa University of Technology, Częstochowa, Poland, 2011.

[2] K. S. Miller and B. Ross, An Introduction to the Fractional Calculus and Fractional Differential Equations, John Wiley \& Sons Inc., New York, NY, USA, 1993.

[3] A. Oustaloup, La Derivation Non Entiere, Hermes, Paris, France, 1995.

[4] J. A. T. Machado, "Analysis and design of fractional-order digital control systems," Systems Analysis Modelling Simulation, vol. 27, no. 2-3, pp. 107-122, 1997.

[5] A. A. Kilbas, H. M. Srivastava, and J. J. Trujillo, Theory and Applications of Fractional Differential Equations, vol. 204, Elsevier Science B.V., Amsterdam, The Netherlands, 2006.

[6] J. T. Machado, V. Kiryakova, and F. Mainardi, "Recent history of fractional calculus," Communications in Nonlinear Science and Numerical Simulation, vol. 16, no. 3, pp. 1140-1153, 2011.

[7] K. Deithelm, N. J. Ford et al., "Analysis of fractional differential equation," Numerical Analysis Report 377, The University of Manchester, 2003. 
[8] R. W. Ibrahim and S. Momani, "On the existence and uniqueness of solutions of a class of fractional differential equations," Journal of Mathematical Analysis and Applications, vol. 334, no. 1, pp. 1-10, 2007.

[9] I. Podlubny, Fractional Differential Equations, vol. 198, Academic Press Inc., San Diego, Calif, USA, 1999.

[10] K. B. Oldham and J. Spanier, The Fractional Calculus, Academic Press, New York. NY, USA, 1974.

[11] S. Momani, "Analytical approximate solution for fractional heat-like and wave-like equations with variable coefficients using the decomposition method," Applied Mathematics and Computation, vol. 165, no. 2, pp. 459-472, 2005.

[12] S. Momani, Z. Odibat, and A. Alawneh, "Variational iteration method for solving the space- and time-fractional KdV equation," Numerical Methods for Partial Differential Equations, vol. 24, no. 1, pp. 262-271, 2008.

[13] Z. Odibat and S. Momani, "The variational iteration method: an efficient scheme for handling fractional partial differential equations in fluid mechanics," Computers $\mathcal{E}$ Mathematics with Applications. An International Journal, vol. 58, no. 11-12, pp. 2199-2208, 2009.

[14] K. Deithelm and A. D. Freed, "The FracPECE subroutine for the numerical solution of differential equations of fractions order," in Forschung und Wiessenschaftliches Rechnen 1998, S. Heinzel and T. Plesser, Eds., no. 52, pp. 57-71, GWDG-Bericht, Gesellschaftfur Wiessenschaftliches Datenverabeitung, Göttingen, Germany, 1999.

[15] K. Deithelm and A. D. Freed, "On the of solution of nonlinear differential equations used in the modeling of viscoplasticity," in Scientific Computing in chemical Engineering-II. Computational Fluid Dynamics Reaction Engineering, and Molecular Properties, F. Keil, W. Mackens, H. Vob, and J. Werther, Eds., pp. 217-224, Springer, Heidelberg, Gemany, 1999.

[16] K. Diethelm, "An algorithm for the numerical solution of differential equations of fractional order," Electronic Transactions on Numerical Analysis, vol. 5, pp. 1-6, 1997.

[17] N. H. Sweilam, M. M. Khader, and R. F. Al-Bar, "Numerical studies for a multi-order fractional differential equation," Physics Letters A, vol. 371, no. 1-2, pp. 26-33, 2007.

[18] G. Micula, T. Fawzy, and Z. Ramadan, "A polynomial spline approximation method for solving system of ordinary differential equations," Babes-Bolyai Cluj-Napoca. Mathematica, vol. 32, no. 4, pp. 55-60, 1987.

[19] M. A. Ramadan, "Spline solutions of first order delay differential equations," Journal of the Egyptian Mathematical Society, vol. 13, no. 1, pp. 7-18, 2005.

[20] M. A. Ramadan, T. S. El-Danaf, and M. N. Sherif, "Numerical solution of fractional differential eqautions using polynomial spline functions," submitted.

[21] Z. Ramadan, "On the numerical solution of a system of third order ordinary differential equations by spline functions," Annales Universitatis Scientiarum Budapestinensis de Rolando Eötvös Nominatae, vol. 19, pp. 155-167, 2000.

[22] J. Munkhammar, "Riemann-Liouville fractional derivatives and the Taylor-Riemann series," U.U.D.M. Project Report 7, Department of Mathematics, Uppsala University, 2004.

[23] S. Momani, O. K. Jaradat, and R. Ibrahim, "Numerical approximations of a dynamic system containing fractional derivatives," Journal of Applied Sciences, vol. 8, no. 6, pp. 1079-1084, 2008. 


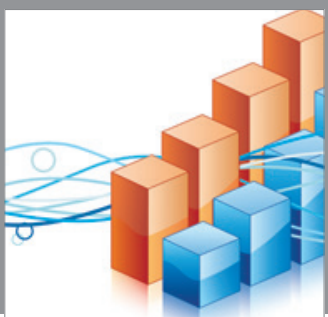

Advances in

Operations Research

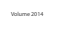

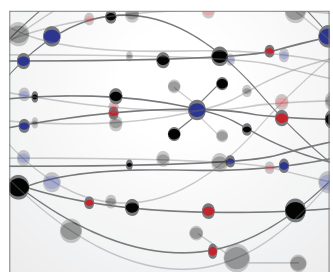

\section{The Scientific} World Journal
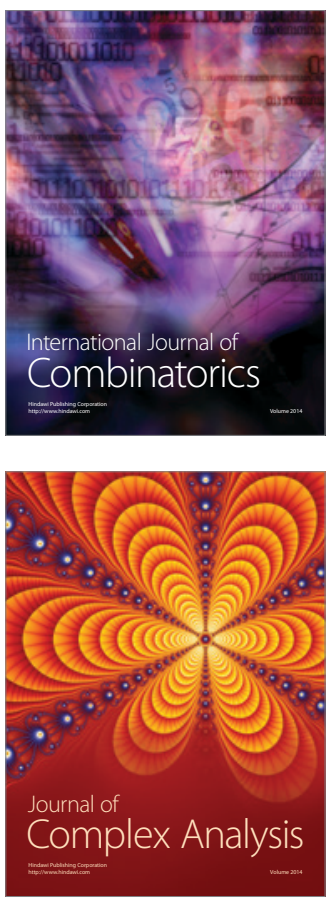

International Journal of

Mathematics and

Mathematical

Sciences
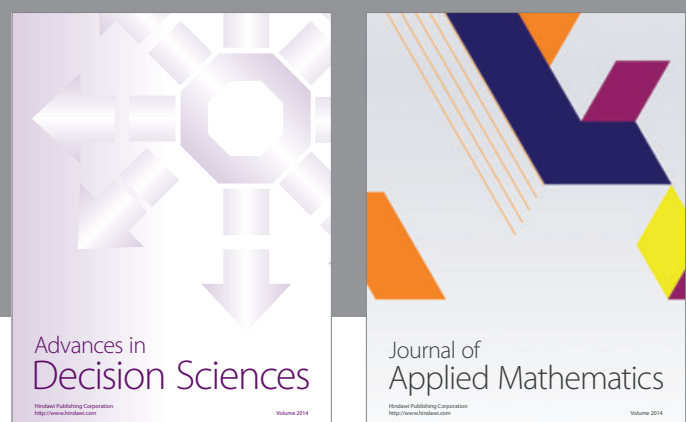

Journal of

Applied Mathematics
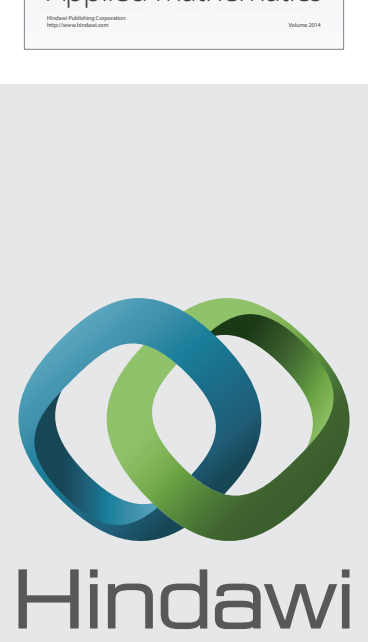

Submit your manuscripts at http://www.hindawi.com
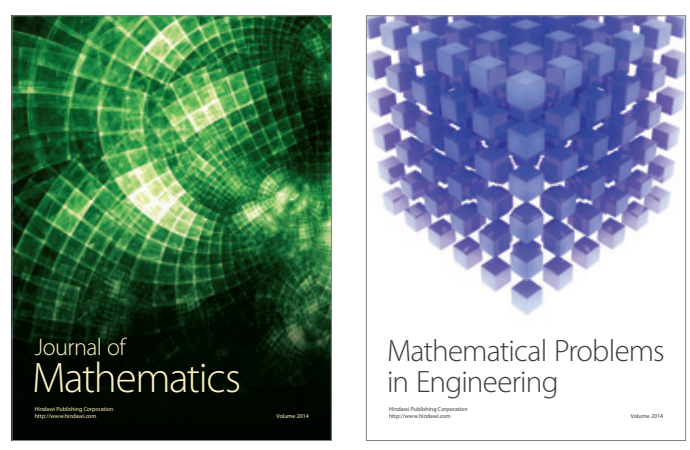

Mathematical Problems in Engineering
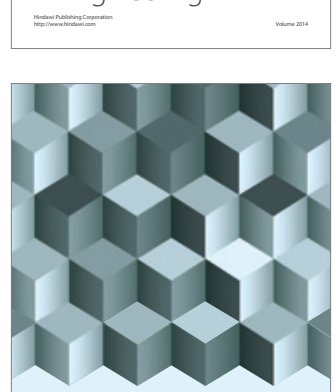

Journal of

Function Spaces
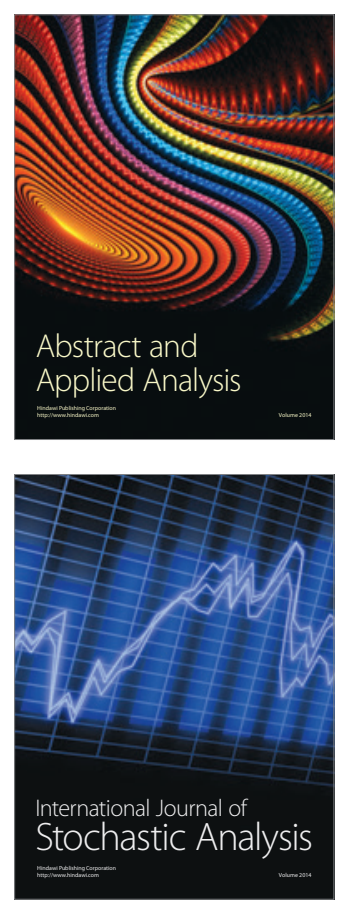

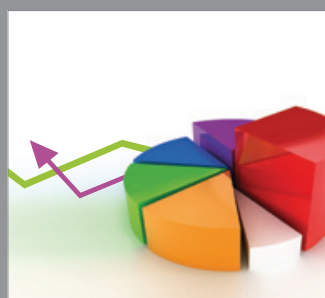

ournal of

Probability and Statistics

Promensencen
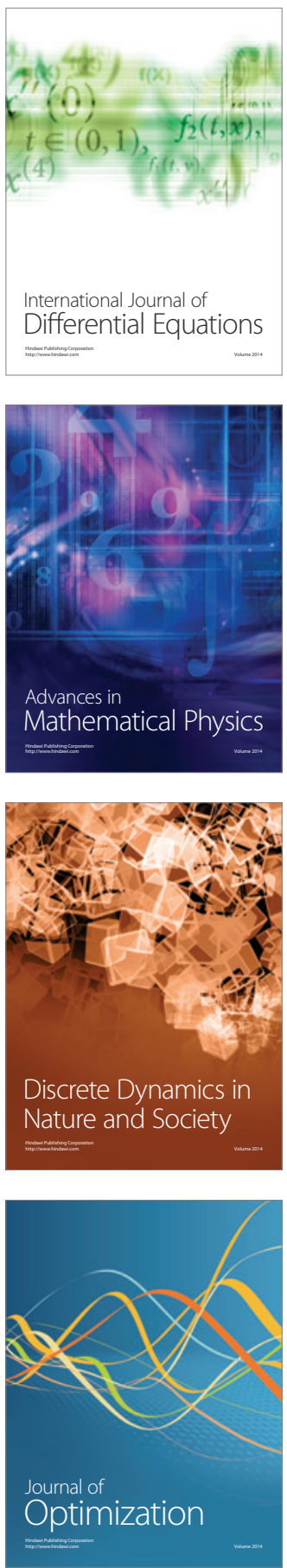